\title{
EXISTENCE AND UNIQUENESS OF SOLUTION OF SOME BOUNDARY VALUE PROBLEMS FOR DELAY DIFFERENTIAL EQUATIONS
}

\author{
Dr. Nedal. Hassan E. ELjaneid ${ }^{1 *}$
}

*1 Department of Mathematics, Faculty of Sciences, University of Tabuk, Kingdom of Saudi Arabia

*Corresponding Author: -

\begin{abstract}
: -
We presented as study to establish the existence and uniqueness of solution some three-point boundary value problems for second order differential equations.
\end{abstract}

Keyword: Boundary Value Problems, Delay Differential Equations

\section{(c) $(2)$ (i)}




\section{INTRODUCTION:}

This paper is concerned with some existence and of solutions for some boundary value problem for Delay differential equations:

$$
\begin{gathered}
\ddot{x}(t)=f\left(t, x(t), \dot{x}(t), x\left(t-\tau_{0}\right), \dot{x}(t-\tau)\right) \\
x(t)=\dot{x}(t)=0, t<0, x(\eta)=\alpha x(1), x(0)=0,0<\eta<1
\end{gathered}
$$

It should be noted that the differential equations have penetrated all branches of contemporary science. The goal is how to use a creative method to study the existence and uniqueness of problem of three-dimension boundary values from the second level of the late nonlinear differential equations. The composition method will be used when dealing with the second problem.

\section{Preliminaries}

\section{1 : Initial Conditions and Boundary conditions:}

In mathematics, in the field of differential equations, a boundary value problem is a differential equation together with a set of additional constraints, called the boundary conditions. A solution to a boundary value problem is a solution to the differential equation which also satisfies the boundary conditions

For example, if the independent variable is time over the domain [0,1], a boundary value problem would specify values for $y(t)$ both $t=0$ and $t=1$, whereas an initial value problem would specify a value of $y(t)$ and $y(t)$ at time $t=0$

\section{2: The contraction mapping:}

A contraction in a metric space is a mapping $f: X \rightarrow X \quad$ such that there exists $0<c<1$ such that for all $x, y \in X, x \neq y$ , it holds that $d(f(x), f(y)) \leq c d(x, y)$. Indeed, the Banach fixed-point theorem shows that such mappings on complete metric spaces always have a unique fixed point.

\section{3 : The fixed point:}

Fixed points of functions in the complex plane commonly lead to beautiful fractal structures. For example, the plots above color the value of the fixed point (left figures) and the number of iterations to reach a fixed point (right figures) for cosine (top) and sine (bottom). Newton's method, which essentially involves a fixed point computation in order to find roots, leads to similar fractals in an analogous way. Points of an autonomous system of ordinary differential equations at which $\frac{d x_{1}}{d t}=f_{1}\left(x_{1}, x_{2} ., x_{n}\right)=0, \ldots, \frac{d x_{n}}{d t}=f_{n}\left(x_{1}, x_{2} . ., x_{n}\right)=0$ are known as fixed points.

\section{Main Results:}

In this part we will prove a single following solution of boundary value problem

$$
\begin{gathered}
\ddot{x}(t)=f\left(t, x(t), \dot{x}(t), x\left(t-\tau_{0}\right), \dot{x}(t-\tau)\right) \\
x(t)=\dot{x}(t)=0, t<0, x(\eta)=\alpha x(1), x(0)=0,0<\eta<1
\end{gathered}
$$

Suppose the operator $f(t, x, y, z, w)$ is define and continues in field

$$
\begin{gathered}
D=\left\{[0,1] \times Q_{1} \times Q_{2} \times Q_{1} \times Q_{2}\right\} \\
Q_{1}=\left\{Z:|Z|_{1} \leq \mathscr{R}_{1}\right\} \quad \& \quad Q_{1}=\left\{w:\left|w_{1}\right| \leq \mathscr{R}_{2}\right\}
\end{gathered}
$$

also $\tau_{0}(t), \tau_{1}(t)$ are positive continues functions in interval $[0,1]$

The equations $t-\tau(t)=0$ have a finite solutions $\lambda i$,

$$
\begin{gathered}
\ddot{x}(t)-k^{2} x(t)=f\left(t, x(t), \dot{x}(t), x\left(t-\tau_{0}\right), \dot{x}(t-\tau)-k^{2} x(t)\right) \\
x(t)=\dot{x}(t)=0, t<0, x(0)=0 x(\eta)=\alpha x(1), 0<\eta<1
\end{gathered}
$$

So, the boundary problem (3.2) $\mathrm{t}$ can be converted by using the method parameters into the equation the integrally equivalent, to find a general solution

$$
\ddot{x}(t)-k^{2} x(t)=0 \Rightarrow x_{h}=c_{1} \cosh k x+c_{2} \sinh k x
$$

Suppose the general solution

$$
x_{p}(t)=u(t) \cosh k x+v(t) \sinh k x
$$

Satisfying the following conditions:

$$
\begin{gathered}
\dot{u}(t) \cosh k t+\dot{v}(t) \sinh k t=0 \\
\dot{u}(t)=f\left(t, x(t), \dot{x}(t), x\left(t-\tau_{0}\right), \dot{x}(t-\tau)-k^{2} x(t)\right)
\end{gathered}
$$

By solving the last two equations we get 


$$
\begin{gathered}
u(t)=\frac{-1}{k} \int_{0}^{t} \sinh k s\left(f\left(t, x(t), \dot{x}(t), x\left(t-\tau_{0}\right), \dot{x}(t-\tau)-k^{2} x(t)\right) d s\right. \\
v(t)=\frac{1}{k} \int_{0}^{t} \cos k s\left[\begin{array}{c}
f\left(t, x(t), \dot{x}(t), x\left(t-\tau_{0}\right), \dot{x}(t-\tau)\right. \\
\left.-k^{2} x(t)\right)
\end{array}\right] d s
\end{gathered}
$$

Substitute in equations (3.4) to (3.5)

$$
x(t)=\frac{1}{k(\sinh k \eta-\alpha \sinh k)}(\sinh k \eta-\alpha \sinh k)
$$

$$
\begin{gathered}
\int_{0}^{t} \sinh k(t-s) k^{2} x(s)-f\left(s, x(s), x(s), x\left(s-\tau_{0}(s), x\left(s-\tau_{1}(s) d s\right.\right.\right. \\
++\alpha \int_{0}^{1} \sinh k t \sin k(1-s)\left[k^{2} x(s)-f\left(s, x(s), x(s), x\left(s-\tau_{0}(s), x\left(s-\tau_{1}(s)\right]\right.\right.\right. \\
\quad-\int_{0}^{n} \sinh k t \sin k(\eta-s)\left[k^{2} x(s)-f\left(s, x(s), x(s), x\left(s-\tau_{0}(s), x(x(s\right.\right.\right. \\
\left.-\tau_{0}(s)\right] d s \\
\int_{0}^{t} \cosh (t-s) k^{2} x(s)-f\left(s, x(s), x(s), x\left(s-\tau_{0}(s), x\left(s-\tau_{1}(s) d s\right.\right.\right. \\
+\alpha \int_{0}^{1} \cosh k t \sin k(1-s)\left[k^{2} x(s)-f\left(s, x(s), x(s), x\left(s-\tau_{0}(s), x\left(s-\tau_{0}(s)\right] d:\right.\right.\right. \\
\quad-\int_{0}^{n} \cosh k t \sin k(\eta-s)\left[k^{2} x(s)-f\left(s, x(s), x(s), x\left(s-\tau_{0}(s), \quad(3.7)\right.\right.\right.
\end{gathered}
$$

Theorem of existence and uniqueness of problem (3.1):

In boundary value problem (3.1), suppose that :

(1) $\frac{\partial f}{\partial z_{1}}, \frac{\partial f}{\partial w_{1}}, \frac{\partial f}{\partial z_{2}}, \frac{\partial f}{\partial w_{2}}$ continuous for every value $t \in[0,1], \forall z_{1}, w_{1}, z_{2}, w_{2}$

(2) $L_{1}, L_{2}, L_{3}$ and $L_{4}$ positive constants

$$
\begin{gathered}
0 \leq \frac{\partial f}{\partial z_{1}} \leq L_{1}, 0 \leq, \frac{\partial f}{\partial w_{1}} \leq L_{2}, 0 \leq \frac{\partial f}{\partial z_{2}} \leq L_{3}, \leq \frac{\partial f}{\partial w_{2}} \leq L_{4}, \forall t \in[0,1] \\
\text { and } \forall z_{1}, w_{1}, z_{2}, w_{2} \\
\text { (3) } \frac{M}{k^{2}}\left|\frac{\sinh k(1+\eta)-\sinh k \eta-\sinh k}{\sinh k-\alpha \sinh k}\right| \leq R_{1} \\
\frac{M}{k}\left|\frac{\cosh k(1+\eta)+\alpha(1-\cosh k)-\cos k}{\sinh k-\alpha \sinh k}\right| \leq R_{2}
\end{gathered}
$$

There is only one solution of problem (1) on condition $\left\|A_{K}\right\|<1$

$$
\begin{aligned}
A_{K}= & {\left[\begin{array}{c}
\frac{\left|k^{2}-L_{1}-L_{3}\right|}{k^{2}}\left(\frac{a}{c}\right) \frac{\left|L_{2}+L_{4}\right|}{k^{2}}\left(\frac{a}{c}\right) \\
\frac{\left|k^{2}-L_{1}-L_{3}\right|}{k}\left(\frac{a}{c}\right) \frac{\left|L_{2}+L_{4}\right|}{k}\left(\frac{a}{c}\right)
\end{array}\right] } \\
& a=|\sinh k(1-\eta)-\sinh k \eta-\sinh k| \\
b & =|\cosh k(1+\eta)+\alpha(1-\cosh k)-\cos k| \\
c & =|\sinh k-\alpha \sinh k|
\end{aligned}
$$

(4) Furthermore it $\forall k: k \geq \max \left(\frac{L_{1}+L_{3}}{2}, \frac{L_{2}+L_{4}}{2}\right)$

$$
\begin{gathered}
x_{0}(t)=0, \dot{x}_{0}(t)=0 \\
x_{m+1}(t)=\frac{1}{k(\sinh k \eta-\alpha \sinh k)}\{(\sinh k \eta-\alpha \sinh k) \\
\int_{0}^{t} \sinh k(t-s)\left[k^{2} x_{m}(s)-f\left(s, x_{m}(s), x_{m}(s), x_{m}\left(s-\tau_{0}(s), x_{m}(s-\right.\right.\right. \\
\left.\tau_{1}(s)\right] d s \quad+\alpha \int_{0}^{1} \sinh k t \sin k(1-s)\left[k^{2} x_{m}(s)-\right. \\
f\left(s, x_{m}(s), x_{m}(s), x_{m}\left(s-\tau_{0}(s), x_{m}\left(\left(s-\tau_{1}(s)\right)\right]\right.\right. \\
-\int_{0}^{n} \sinh k t \sin k(\eta-s)\left[k^{2} x_{m}(s)-f\left(s, x_{m}(s), x_{m}(s), x_{m}\left(s-\tau_{0}(s), x_{m}\left(\left(s-\tau_{1}(s)\right)\right] d s\right.\right.\right.
\end{gathered}
$$




$$
\begin{gathered}
\int_{0}^{t} \sinh k(t-s)\left[k^{2} x_{m}(s)-f\left(s, x_{m}(s), x_{m}(s), x_{m}\left(s-\tau_{0}(s), x_{m}(s-\right.\right.\right. \\
\left.\tau_{1}(s)\right] d s \quad+\alpha \int_{0}^{1} \sinh k t \sin k(1-s)\left[k^{2} x_{m}(s)-\right. \\
f\left(s, x_{m}(s), x_{m}(s), x_{m}\left(s-\tau_{0}(s), x_{m}\left(\left(s-\tau_{1}(s)\right)\right]\right.\right. \\
-\int_{0}^{n} \sinh k t \sin k(\eta-s)\left[k^{2} x_{m}(s)-f\left(s, x_{m}(s), x_{m}(s), x_{m}\left(s-\tau_{0}(s), x_{m}\left(\left(s-\tau_{1}(s)\right)\right] d s\right.\right.\right. \\
x_{m+1}(t)=\frac{1}{(\sinh k \eta-\alpha \sinh k)}\{(\sinh k \eta-\alpha \sinh k) \\
\int_{0}^{t} \cosh k(t-s)\left[k^{2} x_{m}-(s) f\left(s, x(s), x_{m}(s), x_{m}\left(s-\tau_{0}(s), x_{m}\left(s-\tau_{1}(s)\right)\right)\right] d s\right. \\
+\alpha \int_{0}^{1} \cosh k(1-s)\left[k^{2} x_{m}(s)-f\left(s, x_{m}(s), x_{m}(s), x_{m}\left(s-\tau_{0}(s), x_{m}\left(\left(s-\tau_{1}(s\right.\right.\right.\right.\right. \\
-\int_{0}^{n} \cosh k t \sin k(\eta-s)\left[k^{2} x_{m}(s)-f\left(s, x_{m}(s), x_{m}(s), x_{m}\left(s-\tau_{0}(s)\right] d s(3.10)\right.\right.
\end{gathered}
$$

proof: Define the space $C[0,1]$, the opretors $U, V, G$

Suppose the vector space functions $c: y(t)=\left[\begin{array}{l}x(t) \\ \dot{x}(t)\end{array}\right]: x(t), \dot{x}(t)$ are

continuous functions $\|y(t)\|_{c}=\max \left[\begin{array}{l}\max _{0 \leq t \leq 1}\|x(t)\| \\ \max _{0 \leq t \leq 1}\|\dot{x}(t)\|\end{array}\right]$

let operator $\mathrm{c}$ :

$$
\begin{gathered}
G(y(t))=\left\{\frac{1}{k(\sinh k \eta-\alpha \sinh k)} \quad\{\sinh k \eta-\alpha \sinh k)\right. \\
\int_{0}^{t} \sinh k(t-s) k^{2} x(s)-f\left(s, x(s), x(s), x\left(s-\tau_{0}(s), x\left(s-\tau_{1}(s) d s\right.\right.\right. \\
++\alpha \int_{0}^{1} \sinh k t \sin k(1-s)\left[k^{2} x(s)-f\left(s, x(s), x(s), x\left(s-\tau_{0}(s), x\left(s-\tau_{1}(s)\right]\right.\right.\right. \\
-\int_{0}^{n} \sinh k t \sin k(\eta-s)\left[k^{2} x(s)-f\left(s, x(s), x(s), \dot{x}\left(s-\tau_{0}(s), \dot{x}(s\right.\right.\right. \\
\left.\left.\left.-\tau_{1}(s)\right)\right) d s\right\} \\
\left\{\frac{1}{(\sinh k \eta-\alpha \sinh k)}\{(\sinh k \eta-\alpha \sinh k)\right. \\
\int_{0}^{t} \cosh k(t-s) f\left(s, x_{m}(s), x_{m}(s), x_{m}\left(s-\tau_{0}(s), x_{m}\left(s-\tau_{1}(s)\right] d s+\right.\right. \\
\alpha \int_{0}^{1} \cosh k t \sin (1-s)\left[f-k^{2} x_{m}(s)\right] d s \\
\quad-\int_{0}^{n} \cosh k t \sin (\eta-s)\left[f-k^{2} x_{m}(s)\right] d s \quad(3.10)
\end{gathered}
$$

Suppose $y(t) \in \check{S}: \check{S}=\left\{y(t) \in c:\|y(t)\|_{c} \leq \max \left(R_{1}, R_{2}\right)\right\}$

\section{Use condition (3.3):}

and

$$
U(y(t)) \leq \frac{M}{K^{2}} \frac{\sinh k(1+\eta)-\sinh k \eta-\sinh k)}{(\sinh k \eta-\alpha \sinh k)} \leq R_{1}
$$

$$
V(y(t)) \leq \frac{M}{k}\left|\frac{\cosh k(1+\eta)+\alpha(1-\cosh k)-\cos k}{\sinh k-\alpha \sinh k}\right| \leq R_{2}
$$

$G(y(t)) \in \check{S}$

In other words, we find that the operator $G(y(t))$ is transports closed and limited set and convexity $S$ for the same set.

To prove that we only need to prove that two functions $x_{m}(t), x_{m}(t)$ 
Representing the Cauchy sequence

(i) $l m_{+1}(t)=x_{m+1}(t)-x_{m}(t)$

(ii) $h m_{+1}(t)=\dot{x_{m+1}}(t)-\dot{x_{m}}(t)$

(3.11) $(i i i)\left\|\quad l_{m+1}(t)\right\|=\max _{0 \leq t \leq 1}\left|l_{m+1}(t)\right|, m=0,1,2, .$.

(iv) $\left\|h_{m+1}(t)\right\|=\max _{0 \leq t \leq 1}\left|h_{m+1}(t)\right|, m=0,1,2,$.

$$
\begin{aligned}
& l_{m+1}(t)=\frac{1}{k(\sinh k \eta-\alpha \sinh k)}\{\{(\sinh k \eta-\alpha \sinh k) \\
& \left\{\int_{0}^{t} \sinh k(t-s)\left[\left(k^{2}-\frac{\partial f}{\partial z_{1}}-\frac{\partial f}{\partial z_{2}}\right) l_{m}(t)-\left(\frac{\partial f}{\partial w_{1}}-\frac{\partial f}{\partial w_{2}},\right) h_{m}(t)\right\} \mathrm{ds}\right. \\
& +\alpha \int_{0}^{1} \sinh k t \sin k(1-s)\left[k^{2}-\left(k^{2}-\frac{\partial f}{\partial z_{1}}-\frac{\partial f}{\partial z_{2}}\right) l_{m}(t)-\left(\frac{\partial f}{\partial w_{1}}+\left(\frac{\partial f}{\partial w_{2}}\right) h_{m}(t)\right] d s\right. \\
& -\int_{0}^{n} \sinh k t \sin k(\eta-s)\left(k^{2}-\frac{\partial f}{\partial z_{1}}-\frac{\partial f}{\partial z_{2}}\right) l_{m}(t)-\left(\frac{\partial f}{\partial w_{1}}+\left(\frac{\partial f}{\partial w_{2}}\right) h_{m}(t)\right] d s(3.12) \\
& l_{m+1}(t)=\frac{1}{k(\sinh k \eta-\alpha \sinh k)}\{\{(\sinh k \eta-\alpha \sinh k) \\
& \left\{\int_{0}^{t} \cosh k(t-s)\left[\left(k^{2}-\frac{\partial f}{\partial z_{1}}-\frac{\partial f}{\partial z_{2}}\right) l_{m}(t)-\left(\frac{\partial f}{\partial w_{1}}-\frac{\partial f}{\partial w_{2}},\right) h_{m}(t)\right\} \mathrm{ds}\right. \\
& +\alpha \int_{0}^{1} \sinh k t \sin k(1-s)\left[k^{2}-\left(k^{2}-\frac{\partial f}{\partial z_{1}}-\frac{\partial f}{\partial z_{2}}\right) l_{m}(t)-\left(\frac{\partial f}{\partial w_{1}}+\left(\frac{\partial f}{\partial w_{2}}\right) h_{m}(t)\right] d s\right. \\
& -\int_{0}^{n} \cosh k t \sin k(\eta-s)\left(k^{2}-\frac{\partial f}{\partial z_{1}}-\frac{\partial f}{\partial z_{2}}\right) l_{m}(t)-\left(\frac{\partial f}{\partial w_{1}}+\left(\frac{\partial f}{\partial w_{2}}\right) h_{m}(t)\right] d s(3.13) \\
& \text { and whereas } k^{2} \geq \max \left(\frac{L_{1}+L_{3}}{2}, \frac{L_{3}+L_{4}}{2}\right) \text { : } \\
& {\left[\begin{array}{c}
\left\|l_{m+1}(t)\right\| \\
\left\|h_{m+1}(t)\right\|
\end{array}\right] \leq\left[\begin{array}{c}
\frac{\left|k^{2}-L_{1}-L_{3}\right|}{k^{2}}\left(\frac{a}{c}\right) \frac{\left|L_{3}+L_{4}\right|}{k^{2}}\left(\frac{a}{c}\right) \\
\frac{\left|k^{2}-L_{1}-L_{3}\right|}{k^{2}}\left(\frac{a}{c}\right) \frac{\left|L_{3}+L_{4}\right|}{k^{2}}\left(\frac{a}{c}\right)
\end{array}\right]\left[\begin{array}{c}
\left\|l_{m}(t)\right\| \\
\left\|h_{m}(t)\right\|
\end{array}\right]}
\end{aligned}
$$

Equation (3.14) can be written

$$
\begin{aligned}
Z_{m+1} & \leq\left\|A_{k}\right\| Z_{m}, m=1,2, . . \\
\text { or }: Z_{m+1} & \leq\left\|A_{k}^{m}\right\| Z_{1},\left\|A_{k}^{m}\right\| \leq\left\|A_{k}\right\|^{m} \\
Z_{m+1} & \leq\left\|A_{k}^{m}\right\|_{1}
\end{aligned}
$$

If $\|A\| \leq 1 \Rightarrow x_{m}(t), x_{m} \cdot(t)$ is Cauchy sequences in continues function space in interval [0,1] according to the standard defined so exist continuous limit $x(t), \dot{x}(t) \rightarrow x_{m}(t), x_{m}(t)$, and to be two processes limit and integral are commutative so limit of function satisfies two integral equations

To prove uniqueness, suppose that $v(t), u(t)$ are two solutions thus, they satisfy the equations (3.6), (3.7) and with the same analysis that leads to

the equation (3.15): $\|u-v\| \leq A\|u-v\|$, if $\|A\| \leq 1$. It must be in

accordance with the contraction mapping $\|u-v\|=0 \Rightarrow u-v=0 \Rightarrow u=v$

This is the end of proving the existence and uniqueness of solution to boundary problem (3.1)

Example (3.2): Let boundary problem:

$$
\begin{gathered}
x(t)=0.1\left[\frac{x(t)}{4} \sin \mathrm{t}+\dot{\mathrm{x}}(\mathrm{t}-0.5), \mathrm{t} \in[0,1]\right] \\
x(t)=\dot{x}(t)=0, t<0 x(o)=0.2 \text { and } x(0,5)=x(1) \\
f\left(t, y_{1}, z_{1}, y_{2}, z_{2}\right)=0.1\left(\frac{y_{1}}{4} \sin t+z_{2}\right) \text { and let } R_{1}=0.5, R_{2}=2 \\
M=0.210518387, L_{1}=0.022, L_{2}=L_{3}=0, L_{4}=0.1 \\
\left(\begin{array}{cc}
0.0055 & 0.025 \\
0.0055 & 0.025
\end{array}\right) \Rightarrow\|A\|<1
\end{gathered}
$$




$$
\begin{aligned}
& x_{m+1}(t)=0.2-0.1 \int_{0}^{t} S\left[\frac{x_{m}(s)}{4} \sin s+x_{m}^{\cdot}(s-0.5)\right] d s \\
& +\int_{0}^{0.5} t\left[\frac{x_{m}(s)}{4} \sin s+\dot{x_{m}}(s-0.5)\right] d s \\
& +2 t \int_{0.5}^{1}(1-s)\left[\frac{x_{m}(s)}{4} \sin s+x_{m}^{\cdot}(s-0.5)\right] d s \\
& \dot{x}_{m+1}(t)=-0.1 \int_{t}^{0.5}\left[\frac{x_{m}(s)}{4} \sin s+x_{m}(s-0.5)\right] d s \\
& +2 \int_{0.5}^{1}(1-s)\left[\frac{x_{m}(s)}{4} \sin s+\dot{x_{m}}(s-0.5)\right] d s \\
& x_{0}(t)=0.24, x_{0}(t)=1 \\
& y_{1}(t):=0.2-0.00200000000 t^{3}-0.05000000000 t^{2}- \\
& 0.003000000000 t\left(0.25-1 . t^{2}\right)-0.1 t(0.5-1 . t)-0.0260000000 \\
& z_{1}(t):=-0.07675000000+0.003000000000 t^{2}+0.1 t \\
& z_{1}(t) \equiv x_{1}(t) \Rightarrow y_{1} \equiv x_{1} \quad \#
\end{aligned}
$$

\section{Acknowledgment}

Author is grateful to the referee for his/ her valuable comments and suggestions towards the improvement of the paper,

\section{References:}

[1]. Andreson,J.and Thompson ,H.B.Existence of multiple solutions for second order boundary value problems ,.J.Differential Equations ,Vol 166 (2000) .

[2]. Liu, B.Positive solutions of a nonlinear three point boundary value problem , Appl .Math .Comput ,Vol 132 (2002)

[3]. Agarwal, R.P,Philos .Ch.G. and Tsamatos ,P.CH., Global solutions of a singular initial value problem to second order nonlinear delay differential equations, Math compute .Modelling, Vol 43. (2006).

[4]. Yog .H,Liu, Study of singular boundary value problems for second order impulsive differential equations ,J.Math.Anal .Apple ,Vol 331 (2007)

[5]. Agarwal, R.P.O'Regan, Dm and Yan,B,positive solutions of a singular initial value problem to second three point boundary value problems, J.differential equations, Vol 116. (2008).

[6]. Lin, X,Du,Z, and Liu ,W, Uniqueness and existence results for a third order nonlinear multi point boundary value problem ,Apple .Math .Comput, Vol 205 ( 2008 ).

[7]. Kumar,M.and Singh ,N,A collection of computational techniques for solving singular boundary value problems, Adances in Engineering Software, Vol 40 (2009). 